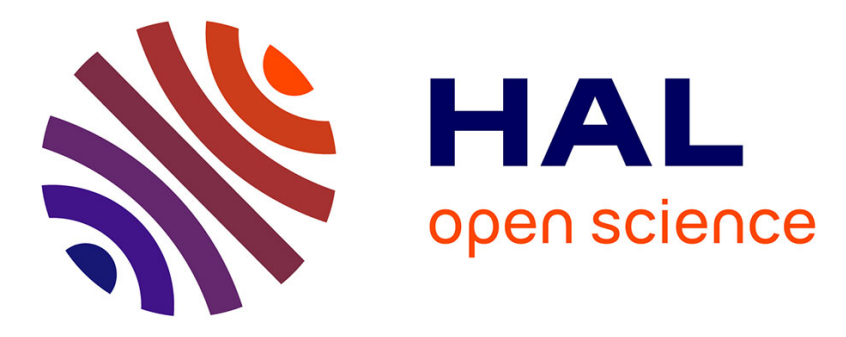

\title{
Empirical Studies in Information Visualization: Seven Scenarios
}

\author{
Heidi Lam, Enrico Bertini, Petra Isenberg, Catherine Plaisant, Sheelagh \\ Carpendale
}

\section{- To cite this version:}

Heidi Lam, Enrico Bertini, Petra Isenberg, Catherine Plaisant, Sheelagh Carpendale. Empirical Studies in Information Visualization: Seven Scenarios. IEEE Transactions on Visualization and Computer Graphics, 2012, 18 (9), pp.1520-1536. 10.1109/TVCG.2011.279 . hal-00932606

\section{HAL Id: hal-00932606 https://hal.inria.fr/hal-00932606}

Submitted on 17 Jan 2014

HAL is a multi-disciplinary open access archive for the deposit and dissemination of scientific research documents, whether they are published or not. The documents may come from teaching and research institutions in France or abroad, or from public or private research centers.
L'archive ouverte pluridisciplinaire HAL, est destinée au dépôt et à la diffusion de documents scientifiques de niveau recherche, publiés ou non, émanant des établissements d'enseignement et de recherche français ou étrangers, des laboratoires publics ou privés. 


\title{
Empirical Studies in Information Visualization: Seven Scenarios
}

\author{
Heidi Lam Enrico Bertini Petra Isenberg Catherine Plaisant Sheelagh Carpendale
}

\begin{abstract}
We take a new, scenario based look at evaluation in information visualization. Our seven scenarios, evaluating visual data analysis and reasoning, evaluating user performance, evaluating user experience, evaluating environments and work practices, evaluating communication through visualization, evaluating visualization algorithms, and evaluating collaborative data analysis were derived through an extensive literature review of over 800 visualization publications. These scenarios distinguish different study goals and types of research questions and are illustrated through example studies. Through this broad survey and the distillation of these scenarios we make two contributions. One, we encapsulate the current practices in the information visualization research community and, two, we provide a different approach to reaching decisions about what might be the most effective evaluation of a given information visualization. Scenarios can be used to choose appropriate research questions and goals and the provided examples can be consulted for guidance on how to design one's own study.
\end{abstract}

Index Terms - Information visualization, evaluation

\section{INTRODUCTION}

Evaluation in information visualization is complex since, for a thorough understanding of a tool, it not only involves assessing the visualizations themselves, but also the complex processes that a tool is meant to support. Examples of such processes are exploratory data analysis and reasoning, communication through visualization, or collaborative data analysis. Researchers and practitioners in the field have long identified many of the challenges faced when planning, conducting, and executing an evaluation of a visualization tool or system [10, 41, 54, 63]. It can be daunting for evaluators to identify the right evaluation questions to ask, to choose the right variables to evaluate, to pick the right tasks, users, or data sets to test, and to pick appropriate evaluation methods. Literature guidelines exists that can help with these problems but they are almost exclusively focused on methods- "structured as an enumeration of methods with focus on how to carry them out, without prescriptive advice for when to choose between them." ([54, p.1 ], author's own emphasis).

This article takes a different approach: instead of focusing on evaluation methods, we provide an in-depth discussion of evaluation scenarios, categorized into those for understanding data analysis processes and those which evaluate visualizations themselves.

The scenarios for understanding data analysis are:

- Heidi Lam is with Google Inc. E-mail: heidi.lam@gmail.com

- Enrico Bertini is with the University of Konstanz E-mail: enrico.bertini@uni-konstanz.de

- Petra Isenberg is with INRIA E-mail: petra.isenberg@inria.fr

- Catherine Plaisant is with the University of Maryland E-mail:plaisant@cs.umd.edu

- Sheelagh Carpendale is with the University of Calgary E-mail: sheelagh@ucalgary.ca
- Understanding Environments and Work Practices (UWP)

- Evaluating Visual Data Analysis and Reasoning (VDAR)

- Evaluating Communication Through Visualization (CTV)

- Evaluating Collaborative Data Analysis (CDA)

The scenarios for understanding visualizations are:

- Evaluating User Performance (UP)

- Evaluating User Experience (UE)

- Evaluating Visualization Algorithms (VA)

Our goal is to provide an overview of different types of evaluation scenarios and to help practitioners in setting the right evaluation goals, picking the right questions to ask, and to consider a variety of methodological alternatives to evaluation for the chosen goals and questions. Our scenarios were derived from a systematic analysis of 850 papers (361 with evaluation) from the information visualization research literature (Section 5). For each evaluation scenario, we list the most common evaluation goals and outputs, evaluation questions, and common approaches in Section 6. We illustrate each scenario with representative published evaluation examples from the information visualization community. In cases where there are gaps in our community's evaluation approaches, we suggest examples from other fields. We strive to provide a wide coverage of the methodology space in our scenarios to offer a diverse set of evaluation options. Yet, the "Methods and Examples" lists in this paper are not meant to be comprehensive as our focus is on choosing among evaluation scenarios. Instead we direct the interested reader towards other excellent overview articles listed in Section 4, which focused on methods.

The major contribution of our work is therefore a new, scenario-based view of evaluations. Our goal is to:

- encourage selection of specific evaluation goals before 
considering methods, by organizing our guide by scenarios rather than by methods;

- broaden the diversity of evaluation methods considered for each scenario, by providing examples from other disciplines in context of evaluation goals commonly found in our community;

- provide an initial step in developing a repository of examples and scenarios as a reference.

\section{The Scope of Evaluation}

In this paper, we take the broad view that evaluation that can occur at different stages of visualization development:

1) Pre-design e.g., to understand potential users' work environment and work flow

2) Design e.g., to scope a visual encoding and interaction design space based on human perception and cognition

3) Prototype e.g., to see if a visualization has achieved its design goals, to see how a prototype compares with the current state-of-the-art systems or techniques

4) Deployment e.g., to see how a visualization influences workflow and its supported processes, to assess the visualization's effectiveness and uses in the field

5) Re-design e. g., to improve a current design by identifying usability problems

Our definition of evaluation is therefore not restricted to the analysis of specific visual representations; it can also focus on a visualization's roles in processes such as data analysis, or on specific environments to which visualizations might be applied. Evaluations in these stages are very relevant, important, and specific to data analysis as previously discussed $[10,41,54,73]$. As it is very in visualization to assess visualization algorithms we extend our notion of evaluation also to these types of systematic assessments which may not always involve participants (Section 6.7).

With this broad view, the outputs of evaluations are also diverse: they may be specific to a visualization to inform design decisions, or more general such as models and theories, perceptual and cognitive modeling from controlled experiments, and development of metrics based on automatic evaluation of visual quality or salience. We highlight these diverse outcomes in more detail in Section 6.

\section{How to Use This Paper}

This paper is meant to shed light on four specific aspects of information visualization evaluation:

1) Choosing a focus: A clear focus is a necessary prerequisite for successful evaluation [15, 22]. We highlight possible evaluation foci within two scenario categories: the visualization itself (e.g., visual encoding) or its supported processes (e.g., exploratory data analysis). We also briefly describe how possible outputs of the evaluation can be used in the visualization development cycle (Section 2).

2) Picking suitable scenarios, goals, and questions: We describe seven scenarios in Section 6 together with possible evaluation foci, outcomes, and questions within the Goals and Outputs and Evaluation Questions sections of each scenario.

3) Considering applicable approaches: Example approaches can be found in the Methods and Examples sub-sections of Section 6. Each scenario is illustrated with examples of published evaluations, which can be used as references for additional details.

4) Creating evaluation design and planned analyses: We list benefits and limitations of each approach within the scenario sections. While we aimed to provide a diverse range of evaluation methods, the lists are not exhaustive and, thus, we encourage creativity in evaluation design starting from and extending the work referenced here.

\section{Related Work}

In this section, we review related work in the areas of evaluation taxonomies, systematic reviews, and evaluation methodologies and best practices.

\subsection{Evaluation Taxonomies}

Others have approached the problem of guiding researchers and practitioners in visualization evaluation by providing a high-level view of available methodologies and methods as taxonomies. The metrics used for classification have been diverse, ranging from research goals, to design and development stages in which the methodologies can be applied, to methods and types of data collected, or to the scope of evaluation. Table 1 summarizes existing taxonomies and their respective foci.

The diversity exhibited in Table 1 reflects the complexity and richness of existing evaluation methodologies and the difficulty in deriving an all encompassing taxonomy. For example, using research goals as a taxonomy axis is challenging because the same evaluation method may be used for different purposes. One example is laboratory-based studies measuring task completion time to compare between interfaces (also known as "head-to-head" comparisons). Such a method can be used to summarize the effectiveness of an interface ("summative") or to inform design ("formative") [2, 22]. Similar arguments apply to classifying methods based on design and development cycles- the same method may be used differently at different stages. For example, observational techniques may be first used in the pre-design stage to gather background information [41], but may also be used post-release to understand how the newly introduced technology affects workflows. Given these difficulties, we decided on a different approach where we based our discussions on commonly encountered evaluation scenarios instead of methods. Across all the papers we examined, we explored how these scenarios relate to evaluation goals and questions (Section 5). Our goal is to encourage an approach to evaluation that is based on evaluation goals and questions instead of methods and to encourage our community to adopt a wider view on the possibilities for evaluation in information visualization. 


\begin{tabular}{|c|c|c|}
\hline Type & Categories & Refs \\
\hline Evaluation goals & $\begin{array}{l}\text { Summative (to summarize the effectiveness of an interface), formative (to } \\
\text { inform design) }\end{array}$ & $\begin{array}{l}\text { Andrews [2], Ellis and } \\
\text { Dix [22] }\end{array}$ \\
\hline Evaluation goals & $\begin{array}{l}\text { Predictive (e.g., to compare design alternatives and compute usability met- } \\
\text { rics), observational (e.g., to understand user behaviour and performance), } \\
\text { participative (e.g., to understand user behaviour, performance, thoughts, and } \\
\text { experience) }\end{array}$ & $\begin{array}{l}\text { Hilbert and Redmiles } \\
{[34]}\end{array}$ \\
\hline $\begin{array}{l}\text { Evaluation } \\
\text { challenges }\end{array}$ & $\begin{array}{l}\text { Quantitative (e.g., types validity: conclusion (types I \& II errors), construct, } \\
\text { external/internal, ecological), qualitative (e.g., subjectivity, sample size, anal- } \\
\text { ysis approaches) }\end{array}$ & Carpendale [10] \\
\hline Research strategies & $\begin{array}{l}\text { Axes (generalizability, precision, realism, concreteness, obtrusiveness) and } \\
\text { research strategies (field, experimental, respondent, theoretical) }\end{array}$ & McGrath [53] \\
\hline Research methods & $\begin{array}{l}\text { Class (e.g., testing, inspection), type (e.g., log file analysis, guideline reviews), } \\
\text { automation type (e.g., none, capture), effort level (e.g., minimal effort, model } \\
\text { development) }\end{array}$ & Ivory and Hearst [42] \\
\hline Design stages & $\begin{array}{l}\text { Nested Process Model with four stages (domain problem characterization, } \\
\text { data/operation abstraction, encoding/interaction technique design, algorithm } \\
\text { design), each with potential threats to validity and methods of validation }\end{array}$ & Munzner [54] \\
\hline Design stages & $\begin{array}{l}\text { Design/development cycle stage associated with evaluation goals ("ex- } \\
\text { ploratory" with "before design", "predictive" with "before implementation", } \\
\text { "formative" with "during implementation", and "summative" with "after } \\
\text { implementation"). Methods are further classified as inspection (by usability } \\
\text { specialists) or testing (by test users). }\end{array}$ & Andrews [2] \\
\hline Design stages & $\begin{array}{l}\text { Planning \& feasibility (e.g., competitor analysis), requirements (e.g., user } \\
\text { surveys), design (e.g., heuristic evaluation), implementation }(e . g ., \text { style guide), } \\
\text { test \& measure (e.g., diagnostic evaluation), and post release (e.g., remote } \\
\text { evaluation) }\end{array}$ & Usability.net [88] \\
\hline Design stages & Concept design, detailed design, implementation, analysis & Kulyk et al. [46] \\
\hline Data and method & $\begin{array}{l}\text { Data collected (qualitative, quantitative), collection method (empirical, analyt- } \\
\text { ical) }\end{array}$ & Barkhuus and Rode [5] \\
\hline Data & Data collected (qualitative, quantitative, mixed-methods) & Creswell [17] \\
\hline Evaluation scope & Work environment, system, components & Thomas and Cook [82] \\
\hline
\end{tabular}

TABLE 1

Taxonomies of evaluation methods and methodologies based on the type of categorization, the main categories themselves, and the corresponding references.

\subsection{Systematic Reviews}

Our work here is closest in spirit to a subtype of systematic review known as narrative review, which is a qualitative approach and describes existing literature using narratives without performing quantitative synthesis of study results [75]. Systematic reviews is itself a type of evaluation method with the purpose to provide snapshots of existing knowledge based on published study results, where "the researcher focuses on formulating general relations among a number of variables of interest" that "hold over some relatively broad range of populations", [53, p. 158]. To the best of our knowledge, two systematic reviews on evaluation methods have been conducted, both counted the number of papers in specific corpora based on the authors' classification scheme.

The first is Barkhuus and Rode's analysis on 24 years of publications in the proceedings of the SIGHCHI Conference on Human Factors in Computing Systems (CHI) [5]. The researchers found that while the proportion of papers with evaluations increased over time, the quality of the evaluation may not have improved, judging from the decreased median number of participants in quantitative studies, an over-reliance on students as participants, and lack of gender-balanced samples. The second is Perer and Shneiderman's analysis of three years of publications in the proceedings of the IEEE Symposium on Information Visualization (InfoVis) and one year of the IEEE Symposium on Visual Analytics Science and Technology (VAST) [57]. In these corpora, the researchers did not find an increase in proportion of papers with evaluation. Similar to Barkhuus and Rode, Perer and Shneiderman also expressed concerns over the quality of evaluation, as most evaluations conducted were controlled studies with non-domain experts as test subjects. Our focus in contrast was to derive a common set of evaluation questions and approaches to ground the development of our scenarios.

\subsection{Evaluation Methodologies and Best Practices}

There exists a large number of publications that reflect upon current practices in visualization evaluation and provide recommendations to improve our status quo. In fact, the BELIV workshop was created as a venue for researchers 
and practitioners to "explore novel evaluation methods, and to structure the knowledge on evaluation in information visualization around a schema, where researchers can easily identify unsolved problems and research gaps" [8]. Providing a complete summary of publications on evaluation probably deserves a paper of its own. In this section, we briefly outline some of the commonly discussed challenges.

Kosara et al. advocated the use of studies in visualization by enumerating situations where and how user studies may be useful [14]. This paper is close to ours in spirit, except we cover a more diverse set of methods and organize evaluation situations into scenarios.

Other publications focus on different aspects of evaluation. In terms of study design, many papers urge researchers to think about the goals of the evaluation [15, 22]. The evaluation goal heavily influences the choice of research strategies, the types of data and methods of collection, and the methods of data analysis. For example, if the goal is to understand how a new technology affects user workflow, then realism is important. In other words, data collection should be from the field using non-intrusive collection mechanisms. Several researchers of these papers that reflect on evaluation commented on the lack of realism in the existing evaluation efforts, which are mostly laboratory based, using basic visual search tasks with non-target users. One way to ensure validity is to ensure realism in tasks, data, workflow, and participants [2, 22, 63]. An alternative is to provide an understanding of situations where some of these requirements can be released, for example, using non-domain expert participants. Other commonly discussed topics of study design include the short durations of most study periods [63], the narrowness of study measurements [63], and possibly insufficient training of participants [2]. In terms of data analysis, concerns have been expressed on the narrowness of questions posed and statistical methods applied [22]. Given that most of the existing evaluation studies are one-offs, researchers have suggested doing follow-up studies to further investigate unanswered questions [22, 47].

In short, all aspects of evaluation require careful attention. This paper is an effort to provide a different kind of guide for visualization researchers and practitioners through concrete scenarios illustrated with existing evaluations.

\section{Methodology}

Early in our project, we decided to take a descriptive rather than a prescriptive approach. In other words, our paper describes and comments on existing practices in evaluating visualizations, but we do not prescribe specific evaluation methods as we believe that the final decision on appropriate methods should be made on a case-by-case basis. We identified seven evaluation scenarios most commonly encountered by visualization researchers which are meant to inform the development of appropriate evaluation strategies. The scenarios were derived from data collected through open coding [17] of publications from four information visualization publication venues (see Table 2). Our approach included the following steps to derive the scenarios:
1-Compiling an evaluation dictionary. Initially, to gather a description of existing evaluation practices in the visualization community, we compiled a dictionary of terms of existing evaluation strategies and techniques and collected matching definitions and example evaluation publications. Our list was compiled based on information solicited by emails to participants of the BELIV 2008 workshop combined with our own knowledge and research (e. g., $[8,10,38,41,47,63])$. The process yielded a wealth of information which required additional structure but provided us with a richer understanding of the types of evaluations commonly used and helped to provide necessary context for us to perform the open coding and tagging of the evaluation papers.

2-Open coding and tagging. From the set of terms and examples collected in the first phase we derived an initial eight tags that classified evaluations in terms of evaluation goals. These tags included topics such as data analysis, decision making, or usability. We selected four major visualization publication venues from which to identify commonly encountered evaluations:

- Eurographics/IEEE Symposium on Visualization (EuroVis)

- IEEE Information Visualization (InfoVis)

- IEEE Visual Analytics Science and Technology (VAST)

- Palgrave's Journal of Information Visualization (IVS)

From these sources, we collected 850 papers and conducted a first coding pass that culled papers that did not mention evaluation and left 361 evaluation papers for further consideration. Publication years and number of papers involved are summarized in Table 2.

Three of us performed the open-coding [17] on parts of the dataset. For each paper, we attached one or more tags from the initial set and recorded the reported evaluation goals and methods. As we proceeded in coding selected publications, each of us independently added new tags to the initial collection, which were then shared among all coders during the tagging period. At regular intervals we discussed the definition of each tag within the group and through consensus, adopted new tags from the other coders during the process and recoded papers with the new tags.

By the end of our publication coding, we had expanded our initial tag set to seventeen tags. Details of the tags can be found in Appendix A.

3-Developing Scenarios. We engaged in one final coding pass during which we grouped tags based on similarity of evaluation goals and research questions. We removed two tags which focused on the development of new evaluation methodologies. We considered these to be beyond the scope of this article as they did not represent actual evaluations which had been conducted. Our final set included 7 tags which represent main distinguishable evaluation questions and goals. This consolidation provides a more manageable list of elements in order to facilitate the applicability of these goals and questions in practice, as described in Section 3. Scenarios, tags, and paper numbers for each are 


\begin{tabular}{cccc}
\hline Venue & Year & Papers & Papers with Eval \\
\hline EuroVis* & $2002-2011$ & 151 & 66 \\
InfoVis & $1995-2010$ & 381 & 178 \\
IVS & $2002-2010$ & 183 & 86 \\
VAST & $2006-2010$ & 123 & 43 \\
\hline Total & & 850 & 361 \\
\hline
\end{tabular}

${ }^{*}$ Pure SciVis papers were excluded from these counts based on visualization type: (e. g. pure volume, molecular, fibre-bundle, or flow visualization).

TABLE 2

Venues included in the open-coding stage to identify commonly encountered evaluation goals, which were then distilled into scenarios. "Paper with Eval" denotes the number of papers which report at least one evaluation.

summarized in Table 3 in the Appendix. The full set of papers with their codes can be found at: http://bit.ly/7-visscenarios. The building of scenarios is, thus, the result of an iterative process among coders where phases of individual grouping and collective consolidation alternated.

\section{Scenarios}

In this section, we present our seven evaluation scenarios which represent classes or categories of evaluations we found in our literature analysis. For each scenario, we provide a definition, identify the popular goals and outputs, the common evaluation questions, and applicable evaluation methods along with concrete examples. Our scenarios can be roughly classified into two broad categories based on their focus. We call these two categories process and visualization. In the process group, the main goal of the evaluation is to understand the underlying process and the roles played by visualizations. While evaluators may record specific user performance and feedback, the goal is to capture a more holistic view of the user experience. In contrast, evaluations can focus on the visualization itself, with the goal to test design decisions, explore a design space, bench-mark against existing systems, or to discover usability issues. Usually in these evaluations, a slice part of the visualization system or technique is tested. We describe the four visualization scenarios in Sections 6.16.4, followed by the three process scenarios in Sections 6.56.7 .

\subsection{Understanding Environments and Work Prac- tices (UWP)}

Evaluations in the UWP group elicit formal requirements for design. In most software development scenarios it is recommended to derive requirements from studying the people for which a tool is being designed [77] but, as noted by Munzner [54, p.7], "hardly any papers devoted solely to analysis at this level [problem characterization] have been published in venues explicitly devoted to visualization." Similarly, Plaisant [63] has argued that there is a growing need for information visualization designers to study the design context for visualization tools including tasks, work environments, and current work practices. Yet, in information visualization research studying people and their task processes is still rarely done and only few notable exceptions have published results of these analyses (e.g., $[40,85])$.

\subsubsection{UWP: Goals and Outputs}

The goal of information visualization evaluations in this category is to work towards understanding the work, analysis, or information processing practices by a given group of people with or without software in use. The output of studies in this category are often design implications based on a more holistic understanding of current workflows and work practices, the conditions of the working environment itself, and potentially current tools in use. Studies that involve the assessment of people's work practices without a specific visualization tool typically have the goal to inform the design of a future visualization tool. Studies involving the assessment of work flow and practices with a specific tool in people's work environment try to assess factors that influence the adoption of a tool to find out how a tool has been appropriated and used in the intended work environments in order to elicit more specific design advice for future versions of the tool.

\subsubsection{UWP: Evaluation questions}

Questions in this scenario usually pertain to identifying a set of features that a potential visualization tool should have. For example:

- What is the context of use of visualizations?

- In which daily activities should the visualization tool be integrated?

- What types of analyses should the visualization tool support?

- What are the characteristics of the identified user group and work environments?

- What data is currently used and what tasks are performed on it?

- What kinds of visualizations are currently in use? How do they help to solve current tasks?

- What challenges and usage barriers can we see for a visualization tool?

\subsubsection{UWP: Methods and Examples}

There is a wealth of methods available for studying work environments and work practices. Most of these rely on qualitative data such as interviews or observational data, audio-visual, or written material:

Field Observation. Observational methods are the most common way to elicit information on current work practices and visualization use. We further describe the goals and basics of this method in Section 6.6. In information visualization, few published examples exist of this type of study, but more have been called for [41]. In a study on automotive engineers Sedlmair et al. [72] observed eight analysis experts at their workplace and derived information 
on domain-specific tool use, why tools were used, and when participants entered in collaborative analysis. The researchers then used the results of the study to derive a set of requirements for the design of data analysis systems for this domain. Both this study and another by Tory et al. [85] combined observation with interviews.

Interviews. There are several types of interviewing techniques that can be useful in this context. Contextual inquiry [36] is a user-centered design method in which people are first observed and then interviewed while engaged in their daily routines within their natural work environment. The researcher tries to interfere as little as possible. Picking the right person to interview is critical in order to gather useful results. Interviews can also be conducted within a lab context. These types of interviews are more common in information visualization: Pretorius and van Wijk interviewed domain experts about their own data to learn how they analyzed state transition graphs [65], Brewer et al. [9] interviewed geovisualization experts to learn about multi-disciplinary science collaboration and how it could be facilitated with collaborative visualization tools.

Laboratory Observation. Observational studies also sometimes occur in laboratory settings in order to allow for more control of the study situation. For example, two studies from the collaborative visualization field looked at how visualizations are used and shared by groups of people and how visualization results are integrated [40, 67]. Both studies presented rich descriptions of how people interacted with visualizations and how these activities could be supported by technology.

\subsection{Evaluating Visual Data Analysis and Reason- ing (VDAR)}

Evaluations in the VDAR group study if and how a visualization tool supports the generation of actionable and relevant knowledge in a domain. In general, VDAR evaluation requires fairly well developed and reliable software.

\subsubsection{VDAR: Goals and Outputs}

The main goal of VDAR evaluation is to assess a visualization tool's ability to support visual analysis and reasoning about data. Outputs are both quantifiable metrics such as the number of insights obtained during analysis (e.g., [69, 70]), or subjective feedback such as opinions on the quality of the data analysis experience (e.g., [74]).

Even though VDAR studies may collect objective participant performance measurements, studies in this category look at how an integrated visualization tool as a whole supports the analytic process, rather than studying an interactive or visual aspect of the tool in isolation. We cover the latter case in our scenario Evaluating User Performance in Section 6.5. Similarly, VDAR is more process-oriented than the identification of usability problems in an interface to refine the prototype, which is covered in Section 6.6. Here, we first focus on the case of a single user. Collaboration is discussed in Section 6.4, Evaluating collaborative data analysis.

\subsubsection{VDAR: Evaluation Questions}

Data analysis and reasoning is a complex and ill-defined process. Our sample questions are inspired by Pirolli and Card's model of an intelligence analysis process [61], considering how a visualization tool supports:

- Data exploration? How does it support processes aimed at seeking information, searching, filtering, and reading and extracting information?

- Knowledge discovery? How does it support the schematization of information or the (re-)analysis of theories?

- Hypothesis generation? How does it support hypothesis generation and interactive examination?

- Decision making? How does it support the communication and application of analysis results?

\subsubsection{VDAR: Methods and Examples}

Studying how a visualization tool may support analysis and reasoning is difficult since analysis processes are typically fluid and people use a large variety of approaches [40]. In addition, the products of an analysis are difficult to standardize and quantify since both the process and its outputs are highly context-sensitive. For these reasons, evaluations in VDAR are typically field studies, mostly in the form of case studies. They strive to be holistic and to achieve realism by studying the tool use in its intended environment with realistic tasks and domain experts. However, we also found experiments in which parts of the analysis process were controlled and studied in a laboratory setting.

In this section, we focus on techniques that individual researchers can use, as opposed to community-wide evaluation efforts such as the Visualization Contest or the Visual Analytics Challenge [16]. The Visual Analytics Challenge provides a useful collection of data sets and analysis problems that can be used in wider VDAR evaluations, and a repository of examples that demonstrate how other tools have been used to analyse the data.

Case Studies. Case studies conducted in VDAR are mostly studies on domain experts interacting with a visualization to answer questions from Section 6.2.2. For example, Trafton et al. conducted an exploratory investigation in the field to answer questions such as "How are complex visualizations used, given the large amount of data they contain?" [86, p. 16]. The researchers recruited three pairs of meteorological forecasters and asked them to prepare a written information brief for a flight. The researchers then open-coded video data to capture the type of visualizations used in various stages of the analysis.

In some cases, researchers collect data over a longer period of time (from weeks to months) with participants working on their own problems in their normal environments. Analysis activities may be captured by automated logging or self-reporting, using for example a diary method [81]. Two examples of long-term case studies in visualization evaluation are: Multi-dimensional In-depth Long-term Case studies (MILCs) [76] and insight-based evaluations [70].

MILC evaluations use multiple techniques such as ob- 
servations, interviews, surveys, and automated logging to assess user performance, interface efficacy, and interface utility [76]. In MILC studies, researchers offer assistance to participants in learning the system, and may improve the systems based on participant feedback. MILC evaluations have been employed, for instance, to evaluate knowledge discovery tools [74] and the integration of statistics and visualization [57]. The main question Seo et al. set out to answer in their MILC case studies using the Hierarchical Clustering Explorer (HCE) was "how do HCE and the rankby-feature framework change the way researchers explore their datasets" [74, p. 313]. To answer this data exploration question, Seo et al. used participatory observations [10, p. 36] and interviews, conducted weekly for a period of four to six weeks, during which time the participants were asked to use the tool in their everyday work.

Insight-based evaluations try to capture insight as "an individual observation about the data by the participant, a unit of discovery" [69, p. 4]. Data collection methods proposed are either the diary method or capturing video using a think-aloud protocol. For example, Saraiya et al. conducted a longitudinal study with biologists and bioinformaticians using real-life microarray data [70]. The goals of the study were to deepen understanding of the visual analytics process, to understand how existing tools were used in analysis, and to test out an evaluation methodology. Data was collected using a diary maintained by the participants to record the analysis process, the insights gained from the data, and which visualization and interaction techniques led to insights, and the successes and frustrations participants experienced with the software tools. Over the course of the study, debriefing meetings were held once every two to three weeks for the researchers to discuss data insights and participants' experience with the tools. Unlike the MILC studies, the researchers did not provide any help with the software tools and did not guide their participants' data analysis in any way to minimize the study's impact on the participants' normal data analysis process.

Laboratory Observation and Interviews Similar to case studies, laboratory observation and interviews are qualitative methods to capture the open-endedness of the data analysis and reasoning processes. For example, Grammel et al. used these methods to study how information visualization novices construct visualizations [23]. Participants, who were students, were given a fictitious data set to look for interesting insights. Since the researchers' focus was on the process of constructing visualizations, rather than evaluating a specific visualization, their study used commercial visualization software (Tablaeu) via a human mediator, both to minimize the effects of the interface and to gain a deeper understanding of the construction process. These observations, as well as follow-up interviews, were open coded to derive abstract models on the construction process and its barriers.

Controlled Experiment. Given the open-ended nature of exploration and the specificity of case studies, it may be beneficial to isolate important factors in the analysis process and study them using laboratory experiments. For example, the Scented Widgets study measured how social navigation cues implemented as scents affected information foraging behaviors. Rather than capturing all aspects of VDAR as in case studies, the researchers encapsulated participant behaviors in a few metrics: the number of revisits, the number of unique discoveries, and subjective preferences based on log data [92].

In some cases, experimenters may use the controlled experiment method as part of their evaluation methods to study VDAR. One example is an early insight-based evaluation [69]. ${ }^{1}$ To attain the goals of measuring selected visualization tools' ability to generate insight, the study used a protocol that combined elements of the controlled experiment and usability testing methods. The basic structure of the evaluation was a 3 (datasets) $\times 5$ (visualization tool) between-subjects design. Given the goals to identify and understand insight occurrence, this evaluation collected a rich set of qualitative data using usability testing techniques. For example, a think-aloud protocol was used to solicit participants' observations, inferences, and conclusions about the data set; a diary method to record participants' estimations of potential insights attainable; open coding of video recordings to capture and characterize individual occurrences of insights by domain experts.

Another example is an evaluation of four visual analytics systems, including two versions of Jigsaw [44]. Non-expert participants were asked to identify a hidden terrorist plot using 50 documents viewed with one of the four systems. In addition to scoring accuracy of the answers, Kang et al. also analyzed participants' activity patterns such as document viewing, querying, and note taking. These patterns revealed participants' investigative strategies, how Jigsaw influenced these strategies, as well as their sense-making processes.

\subsection{Evaluating Communication through Visualiza- tion (CTV)}

Evaluations in the CTV group study if and how communication can be supported by visualization. Communication can pertain to aspects such as learning, teaching, and idea presentation as well as casual consumption of visual information as in ambient displays.

\subsubsection{CTV: Goals and Outputs}

Visualizations evaluated as part of this scenario typically have the goal or purpose to convey a message to one or more persons, in contrast to targeting focused data exploration or discovery. Their effectiveness is usually measured in terms of how effectively such a message is delivered and acquired. Ambient displays are a common example, as they are usually built to quickly communicate peripheral information to passers-by.

\subsubsection{CTV: Evaluation questions}

Studies in CTV are often intended to quantify a tool's quality through metrics such as learning rate, information

1. This was an earlier iteration of insight-based evaluation, different from the longitudinal study mentioned above 
retention and accuracy, or qualitative metrics such as interaction patterns of the way people absorb information or approach the tool. Questions thus pertain to the quality with which information is acquired and the modalities with which people interact with the visualizations. Examples are:

- Do people learn better and/or faster using the visualization tool?

- Is the tool helpful in explaining and communicating concepts to third parties?

- How do people interact with visualizations installed in public areas? Are they used and/or useful?

- Can useful information be extracted from a casual information visualization?

\subsubsection{CTV: Methods and Examples}

Controlled Experiments. Quantitative studies aiming at measuring improvement in communication or learning, employ traditional controlled experiment schemes. As an example, Sedig et al. studied how students used a mathematical visualization tool aimed at teaching basic concepts in geometry [71]. A similar study was performed in the context of a basic programming class, using a tool that visualized the role of variables in program animation [68]. This last experiment is of special interest as it highlights how measuring learning may require the study to span several weeks or months and may, thus, take longer than other traditional evaluations.

Field Observation and Interviews. Qualitative methods like direct observation and interviews are often paired up with experiments in this context. The studies mentioned above, for instance, both complement their quantitative approach with observations of tools in use to understand how information is acquired and to better investigate the process that leads to concept learning. In the context of casual visualizations, that is, visualizations that "depict personally meaningful information in visual ways that support everyday users in both everyday work and nonwork situations" [64], direct observation and interviews are common evaluation techniques. For example, Skog et al. [78] study the use of an ambient visualization to convey real-time information of bus departure times in a public university area. The evaluation consists of interviewing people and spending enough time in the area to understand people's interaction with the system. Viegas et al. [89], studied a visual installation in a museum's gallery. The authors observed the reactions of people to the installation and collected people's impressions to draw conclusions on the design. In a similar context, Hinrichs et al. [35] used an observational and an open-coding approach to analyze how visitors in an art museum approached and interacted with a visualization installation. From this, design considerations for information visualizations in the museum context were derived. As noted by Pousman et al. [64], this kind of observational evaluation is often needed in such contexts because it is necessary to capture data in a setting where people use the tools naturally.

\subsection{Evaluating Collaborative Data Analysis (CDA)}

Evaluations in the CDA group study whether a tool allows for collaboration, collaborative analysis and/or collaborative decision making processes. Collaborative data analysis differs from single-user analysis in that a group of people share the data analysis experience and often have the goal to arrive at a joint conclusion or discovery.

\subsubsection{CDA: Goals and Outputs}

Evaluations in this group study how an information visualization tool supports collaborative analysis and/or collaborative decision making processes. Collaborative systems should support both taskwork, the actions required to complete the task, and teamwork, the actions required to complete the task as a group [59]. For collaborative visualization this means that systems must not only support group work well, but also be good data analysis tools (taskwork). We cover the evaluation of taskwork and its questions in other scenarios and focus on teamwork here.

Studies in CDA have varying goals and, thus, are defined by different types of outputs. Most commonly CDA studies aim to gain a more holistic understanding of group work processes or tool use during collaboration with the goal to derive concrete design implications. It is recognized that the study of teamwork is difficult due to a number of factors including a greater number of variables to consider, the complicated logistics of evaluation, or the need to understand and judge group work processes [55]. Collaborative systems (or groupware) can be evaluated on a number of different levels such as the organization it will be embedded in, the team or group that will be using it, or the system itself. While there have been a number of papers concerned with the evaluation of groupware, only few examples of evaluations for collaborative information visualization systems exist.

\subsubsection{CDA: Evaluation Questions}

For the evaluation of collaborative visualization systems the following questions may be relevant:

- Does the tool support effective and efficient collaborative data analysis?

- Does the tool satisfactorily support or stimulate group analysis or sensemaking?

- Does the tool support group insight? [80]

- Is social exchange around and communication about the data facilitated?

- How is the collaborative visualization system used?

- How are certain system features used during collaborative work? What are patterns of system use?

- What is the process of collaborative analysis? What are users' requirements?

\subsubsection{CDA: Methods and Examples}

As research on collaborative visualization systems has only recently begun to receive increased research attention, there are only few examples of studies in this area. We thus draw on results from both Computer-Supported Cooperative 
Work (CSCW) as well as the small set of recent studies in collaborative visualization.

Within the field of CSCW a multitude of study and data collection methods have been applied to the analysis of group work $[55,58]$. The context of group work (e. g., group configuration, work environment) has been identified as a critical factor in the evaluation and acceptance of collaborative systems (e.g., [27, 55, 87]). Yet, several research papers have outlined the practicality of early formative evaluations in less authentic environments (e. g., $[60,87])$. Coupled with later more situated fieldwork a clearer picture of collaborative systems in use and their influence on groups and organizations can be won. Here we highlight a number of possible evaluation techniques.

Heuristic Evaluation. Heuristic evaluation has been previously proposed for the evaluation of visualization systems $[83,95]$. Finding an appropriate set of heuristics is the main challenge for visualization systems not only to evaluate taskwork [95]. For the evaluation of teamwork a set of heuristics for the assessment of effectiveness and efficiency of collaboration has been proposed [4]. These heuristics are based on the mechanics of collaboration [28, 60] or lowlevel actions and interactions that a collaborative system must support in order for group members to be able to complete a task in a shared manner. Other sets include heuristics based on the locales framework to study the influences of locales (places) on social activities [25] or awareness [19].

Log Analysis. Analysis of logs and user traces were the main sources of information analyzed in studies of distributed collaborative web-based information visualization tools $[33,90]$. Both analyses resulted in descriptions and statistics of collaborative use of system features and suggestions for system improvement. Studies involving the investigation of logs or comments have the advantage of being relatively easy to conduct and evaluate. Little interaction with participants is used to analyze specific system features or tool use overall. To elicit more userspecific data these evaluations have been combined with questionnaires or interviews (e. g., [33]). On the other hand, these studies cannot clearly evaluate interaction between participants, their work or other processes that do not generate a traceable log entry.

Field or Laboratory Observation. Qualitative user studies have a long tradition within CSCW [26, 55]. Observational studies are often combined with logging of user activity, questionnaires, or interviews. For example, two recent studies on co-located synchronous collaboration $[39,50]$ used such a combination of techniques to analyze group work and analysis processes. Effectiveness of group work was assessed in the first study by using a scoring mechanism from the VAST contest [16, 39]. Effectiveness of group work is often not well represented by time and accuracy which are common metrics for usability studies. Isenberg et al. studied how effectively their collaborative social network analysis system CoCoNutTrix [38] supported the collaborative analysis process. They performed an observational study and post-session interview to assess how well the system supported the following factors of the collaboration: explicit communication, consequential communication, group awareness, coordination of actions, group insight, subjective work preferences, and general user reactions to the collaborative environment. Also, previous studies exist which have also used timing information to assess group work effectiveness [52].

Without digital systems, other more exploratory observational studies in visualization and visual analytics assessed group analysis processes [40] or collaborative information synthesis [67]. For collaborative systems studies of work processes are often seen as important prerequisites for estimating outcomes of tool use and to develop mature CSCW tools [55].

In contrast to single user systems, collaborative visual analysis systems must also consider the groups interactions and possible harmony/dis-harmony as they proceed in their joint discovery efforts. Stahl [80] defines the notion of group cognition as "computer-supported collaborative knowledge building" and recommends the study of this collaborative knowledge building through discourse analysis and observation. It would be interesting to combine this approach with insight-based methodologies (e. g., [70]) for the study of group insight.

\subsection{Evaluating User Performance (UP)}

Evaluations in the UP group study if and how specific features affect objectively measurable user performance.

\subsubsection{UP: Goals and Outputs}

User performance is predominantly measured in terms of objectively measurable metrics such as time and error rate, yet it is also possible to measure subjective performance such as work quality as long as the metrics can be objectively assessed. The most commonly used metrics are task completion time and task accuracy. Outputs are generally numerical values analyzed using descriptive statistics (such as mean, median, standard deviations, and confidence intervals) and modeled by such methods as ANalysis Of VAriance (ANOVA) to partition observed variance into components.

\subsubsection{UP: Evaluation questions}

Questions addressed using evaluation methods in the UP group are generally narrow and determined prior to the start of the evaluation. There are basically two types of questions:

- What are the limits of human visual perception and cognition for specific kinds of visual encoding or interaction techniques?

- How does one visualization or interaction technique compare to another as measured by human performance?

\subsubsection{UP: Methods and Examples}

Controlled experiments. In order to answer evaluation questions with quantitative and statistically significant results, evaluations in the UP group require high precision. 
The most commonly used methodologies involve an experimental design with only a small number of variables changed between experiment conditions such that the impact of each variable can be measured ([10, p. 28]; [53, p. 156]). Methods are commonly referred to as controlled experiments, quantitative evaluation, or factorial design experiments. A controlled experiment requires the abstraction of real-life tasks to simple tasks that can be performed by a large number of participants repeatedly in each study session [63]. Due to the need of a relatively large number of participants, researchers often need to recruit non-experts. As a result, study tasks have to be further abstracted to avoid the need for domain knowledge. Both types of task abstractions may sacrifice realism. One reason to study human perceptual and cognitive limits is to explore the design space for visualization and interaction techniques. The outcomes of these studies are usually design guidelines, and in some cases, models. For example, Tory et al. explored the design space of point displays and information landscape displays, dimensionality, and coloring method to display spatialized data [84]. Bartram et al. explored the design space of using motion as a display dimension [6]. Heer and Robertson explored the use of animated transitions in linking common statistical data graphics [32].

Another reason to study human perceptual limits is to find out how people perform with specific visualization techniques under different circumstances such as data set sizes and display formats. The goal of the evaluation is to explore the scalability of particular visualization techniques. For example, Yost and North investigated the perceptual scalability of different visualizations using either a 2megapixel display or with data scaled up using a 32 megapixel tiled display [94]. Another example is Lam et al.'s study to assess effects of image transformation such as scaling, rotation and fisheye on visual memory [48]. In some cases, these experiments can be performed outside of laboratories. An increasingly popular approach is crowdsourcing using Amazon's Mechanical Turk service (http://aws.amazon.com/mturk/). Interested readers are directed to validation studies of the method [30, 45].

The second main evaluation goal in UP is to benchmark a novel system or technique with existing counterparts. These are sometimes known as head-to-head comparisons as participants perform the same tasks on all study interfaces. Interface effectiveness is usually defined by objective measurements such as time and accuracy. One example includes the SpaceTree study, where a novel tree browser was compared with a hyperbolic tree browser and an Explorer-type interface which displayed tree data for a number of node-finding and navigation tasks [62].

While study metrics are most commonly time and accuracy, researchers are starting to look at different metrics. One example is memorability. Examples include a study on spatial location memory using Data Mountain in the short term [66], and six months later [18]. In cases where quality of work instead of objective measures are used as metrics, expert evaluators are required. One example is Hornbæk and Frokjær's study on document visualization, where authors of the documents were asked to determine quality of essays produced by participants [37]. Individual differences may also play a role in user performance [11]. For example, in the evaluation of LifeLines, Alonso et al. looked at the interaction between participants' spatial visualization ability and display format (LifeLines vs. Tabular) in displaying temporal personal history information [1].

Field Logs. Systems can automatically capture logs of users interacting with a visualization. Evaluators analyze these logs to draw usage statistics or single out interesting behaviors for detailed study. This kind of evaluation, especially when performed in web-based environments, has the advantage of providing a large number of observations for evaluation. Also, participants can work in their own settings while data is collected, thus providing a type of ecological validity. Two recent works used log-based evaluation. Mackinlay et al. [49] used computer logs to evaluate the visual effectiveness of a function inserted into Tableau to suggest users' predefined visual configurations for the data at hand. Viegas et al. [90] examined how their design decisions for ManyEyes were received after deployment.

\subsection{Evaluating User Experience (UE)}

Evaluations in the UE group study people's subjective feedback and opinions in written or spoken form, both solicited and unsolicited.

\subsubsection{UE: Goals and Outputs}

Evaluation of user experience seeks to understand how people react to a visualization either in a short or a long time span. A visualization here may interchangeably be intended as an initial design sketch, a working prototype, as well as a finished product. The goal is to understand to what extent the visualization supports the intended tasks as seen from the participants' eyes and to probe for requirements and needs. Evaluations in UE produce subjective results in that what is observed, collected, or measured is the result of subjective user responses. Nonetheless objective user experience measurements exist, for example, recording user reactions through the use of body sensors or similar means [51]. Interestingly, several subjective measures simply mirror the measures we have in user performance, with the difference that they are recorded as they are perceived by the participant. Examples are: perceived effectiveness, perceived efficiency, perceived correctness. Other measures include satisfaction, trust, and features liked/disliked, etc. The data collected in such a study can help designers to uncover gaps in functionality and limitations in the way the interface or visualization is designed, as well as uncover promising directions to strengthen the system. In contrast to UP (Evaluating User Performance, Section 6.5), the goal of UE is to collect user reactions to the visualization to inform design. Traditionally, studies in UP are more geared towards the production of generalizable and reproducible results whereas those in UE tend to be specific to the given design problem. While VDAR (Evaluating Visual Data 
Analysis and Reasoning, Section 6.2) focuses on the output generated through the data analysis and reasoning process, UE looks more at the personal experience. UWP (Understanding Environments and Work Practices, Section 6.1) is similar to UE in that prolonged user observation may take place. Nonetheless, UWP focuses on studying users and their environment whereas UE focuses on a specific visualization.

\subsubsection{UE: Evaluation Questions}

The main question addressed by UE is: "what do my target users think of the visualization?" More specifically:

1) What features are seen as useful?

2) What features are missing?

3) How can features be reworked to improve the supported work processes?

4) Are there limitations of the current system which would hinder its adoption?

5) Is the tool understandable and can it be learned?

\subsubsection{UE: Methods and Examples}

Evaluations in this category can take varied forms: they can focus on understanding a small number of users' initial reactions, perhaps in depth (as in case studies) but they can also collect extensive qualitative feedback with statistical relevance, for example, in the form of questionnaires. Evaluations can be short-term to assess current or potential usage and long-term to assess the adoption of a visualization in a real usage scenario. The output consists of data recorded either during or after visualization use. The data can be the result of indirect expert collection of user experience, when the evaluator takes notes on observed behaviors, or of direct user feedback when methods like structured interviews and questionnaires are used.

Informal Evaluation. An informal user feedback evaluation is performed by demoing the visualization to a group of people, often and preferably domain experts, letting them "play" with the system and/or observe typical system features as shown by representatives. The method is characterized by a very limited degree of formalism. For instance, it generally does not have a predefined list of tasks or a structured evaluation script as in usability tests. It is the simplest kind of evaluation and it is, probably for this reason, extremely common. These types of evaluations have been used to: assess "intuitiveness and functionality" [43], "probe for utility and usability" [21], "identify design flaws and users' subjective preferences" [79], "evaluate and improve [our] implementation of the ideas" [20], or "to solicit ideas for improvements and enhancements" [91].

Usability Test. A usability test is carried out by observing how participants perform a set of predefined tasks. For each session, the evaluators take notes of interesting observed behaviors, remarks voiced by the participant, and major problems in interaction. The set of tasks is usually defined to address a subset of features the designer deems important for the project. What differentiates this method from the others is the careful preparation of tasks and feedback material like questionnaires and interview scripts. Its main goal is to perfect the design by spotting major flaws and deficiencies in existing prototypes [24], nonetheless it can also serve the purpose of eliciting overlooked requirements. Wongsuphasawat and Shneiderman [93] ran a well-structured usability test with 8 participants to evaluate Similan, a data visualization tool for the analysis of temporal categorical records. They ran the study with the goal to "examine the learnability", "assess the benefits", "determine if users could understand", and to "observe the strategies the users chose and what problems they encountered while using the tool".

Field Observation. This method is similar to a usability test in that careful observation of users is involved. The observation however happens in a real world setting, where the system under study is used freely. The main goal of field observations is to understand how users interact with the tool in a real setting and thus to derive useful information on how it can be perfected. Often, the information extracted from this kind of study is a series of emergent patterns that can inspire new designs or improve the current one. Sometimes, this kind of study can be followed by a more formal step of questionnaires or interviews to better understand the nature of the observed patterns. An example of field observation is the study of Vizster, a visualization for the exploration of on-line communities [31], where the authors observed usage in an "installation at a large party" where participants were free to use the developed tool.

Laboratory Questionnaire. The large majority of controlled experiments are followed by a subjective user experiment rating phase where participants fill out a questionnaire to solicit their opinions and reactions to the tested visualization. These questions may be expressed in a five- or seven-point Likert Scale, or open-ended with free answers. While this phase of the evaluation is generally coupled with evaluating user experiment studies, we include it here as the method can be used alone. See Section 6.5 for examples of controlled experiments.

\subsection{Evaluating Visualization Algorithms (VA)}

Evaluations in the VA group study the performance and quality of visualization algorithms by judging the generated output quantitatively. A visualization algorithm is broadly defined as a procedure that optimizes the visual display of information according to a given visualization goal.

\subsubsection{VA: Goals and Outputs}

Evaluations of visualization algorithms aim at: (1) showing how a given solution scores in comparison to selected alternatives; (2) exploring limits and behavior of the algorithm according to data size, data complexity, special cases, etc. Algorithm evaluation normally targets performance or visualization quality. Performance is stated in terms of computational efficiency, visualization quality is computed through the definition of a number of metrics. 


\subsubsection{VA: Evaluation questions}

Questions in this scenario usually pertain to the visual effectiveness or computational efficiency with which data is represented. Typical questions in this domain are:

1) Which algorithm shows the patterns of interest better?

2) Which algorithm provides a more truthful representation of the underlying data?

3) Which algorithm produces the least cluttered view?

4) Is the algorithm faster than other state of the art techniques? Under what circumstances?

5) How does the algorithm scale to different data sizes and complexities?

6) How does the algorithm work in extreme cases?

\subsubsection{VA: Methods and Examples}

Within this class we found two main classes of methods: visualization quality assessment and algorithmic performance.

Visualization Quality Assessment. Many of the algorithms employed in visualization use non-trivial procedures to generate views optimized according to a stated visualization goal. In order to assess their effectiveness researchers have used automatic procedures which compare one solution to another. The outcome of such an assessment might be proof of a competitive algorithm or information on variations of the algorithm (e.g., alternative parameter settings). Visualization quality assessment is based on the definition of one or more image quality measures that capture the effectiveness of visual output according to a desired property of the visualization.

Hao et al. [29] used metrics to compare different solutions to dynamic visualization measuring constancy of display and usage of display space for a data stream monitoring tool. Constancy is measured in terms of changed pixels over time and display space in terms of used pixels in the available space. Bederson et al. [7] compared alternative ordered TreeMap algorithms in terms of "the average aspect ratio of a TreeMap layout, and the layout distance change function, which quantify the visual problems created by poor layouts" and provide specific metrics for their computation. Chen et al. [12] compared alternative strategies to construct overview Dendrogram trees by comparing them through an abstraction quality measure. Chen et al. [13] proposed a novel projection technique to visualize large document corpora and compare its effectiveness to state of the art algorithms like PLSA+PE, LSP, ISOMAP, MDS and PCA. They used label prediction accuracy as a quality measure. Documents are labeled by a majority voting procedure and accuracy is measured in relation to the extent by which documents with the same label are located together while documents with different labels are located far away from each other in the visualization space.

Algorithmic Performance. The analysis of algorithmic performance is so common in the whole domain of Computer Science that a full discussion of its features is beyond the scope of the paper. Information visualization, by employing algorithms to display data in clever and

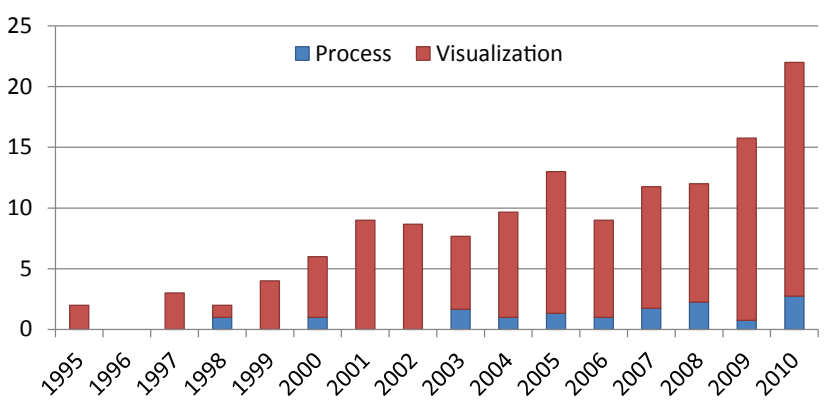

Fig. 1. Each year shows the average number of evaluations coded in each category. Bar height indicates an increase in number of evaluations reported overall.

efficient ways, is of course also often evaluated in terms of algorithmic efficiency as well as visual output. Good evaluations of algorithm performance use an experimental setup in which alternative algorithms score on variations of test data sets and parameters of interest (e.g., data size and complexity). For instance, Artero et al. [3] evaluate alternative algorithms to uncover clusters in parallel coordinates. Peng et al. [56] test alternative heuristic search strategies for their axes reordering procedures and run a number of experiments to see how their behavior changes on different data sizes. Researchers might also use standard benchmarking data sets, where available. One notable example is the graph data sets from the AT\&T Graph Library (www.graphdrawing.org) which is used to evaluate graphdrawing algorithms.

\section{Discussion}

Evaluation is becoming increasingly important in the field of information visualization. The scenarios presented in this paper provide an overview of the wide range of evaluation goals and questions in which the information visualization research community is currently engaged. In this project, we analyzed several hundred evaluation papers (Table 2) and surveyed existing evaluation taxonomies and guideline papers (Table 1). In this section, we discuss evaluation trends we observed from our analysis, as well as our thoughts about evaluation guides.

\subsection{Trends in Evaluation}

The most obvious trend we observed showed that, over the years, evaluations have become more and more prevalent, as seen in the continued increase in the percentage of papers reporting at least one evaluation (Figure 1). The diversity of evaluation scenarios also increased with time, as seen in Figure 2. Nonetheless, the distributions of papers across the seven scenarios remain skewed towards three scenarios: Evaluating User Performance-UP (33\%), Evaluating User Experience-UE (34\%), and Evaluating Visualization Algorithms-VA (22\%). Together, these three scenarios contribute to $85 \%$ of all evaluation papers over the years. This is in sharp contrast to the $15 \%$ share of the process scenarios. 


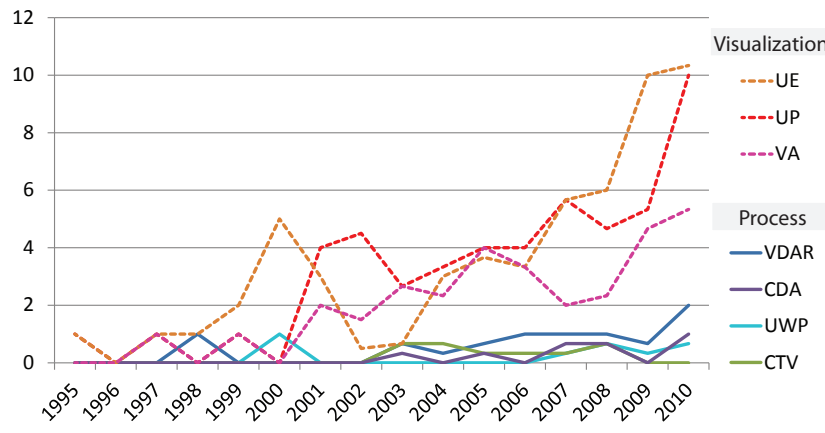

Fig. 2. Each year shows the average number of evaluations coded per scenario per venue.

The fact that the process visualization group is much less represented in the literature is somewhat surprising as the questions in these groups are of high relevance to the field: how can visualization tools be integrated and used in everyday work environments (UWP), how are tasks such as reasoning, knowledge discovery, or decision making supported (VDAR), how does a visualization support communication and knowledge transfer (CTV), and how does a visualization support collaborative analysis (CDA). These questions are of high practical value beyond specific individual tools and can benefit both researchers and practitioners in all areas of visualization.

Several reasons could explain our current evaluation focus. Evaluation in the information visualization community has been following the traditions of Human-Computer Interaction (HCI) and Computer Graphics (CG), both of which also have traditionally focused on controlled experiments, usability evaluations, and algorithm evaluations [24]. Possible questions include: (1) are experiments in the process group simply not being conducted as frequently? (2) does the fact that these types of evaluations are often lengthy requiring field studies, case studies, and extensive qualitative data analysis contribute to their under representation? (3) are we as a community less welcoming to these different-often qualitative-types of evaluations? The lack of evaluations in this group raises questions about whether we as a community should take steps to encourage more evaluations in these groups to be conducted and published.

In the wider HCI community it is comparatively common that publications solely focus on evaluation, often using field and long-term evaluation approaches. In the process evaluation group we used examples from venues outside of the four publication venues we coded to illustrate scenarios in which these types of methodologies are more common (e. g., [40, 57, 86, 87]). As our community continues to grow we need to think critically about what types of evaluations we would like to see more of and how they can benefit our community.

\subsection{Reflecting on this project}

While researching for this project, we analyzed a vast number of publications on evaluation (Table 1). From this analysis, we took a different approach to offer a different type of help to visualization evaluators. The main characteristics of our approach include our focus on goals and outputs rather than methods, offering a wide range of examples in our scenarios, and being descriptive rather than prescriptive.

\subsubsection{Focusing on Goals and Outputs, not Methods}

Instead of focusing on evaluation methods, we focused on evaluation goals and outputs organized as scenarios. We took this approach because we believe that focusing on evaluation methods may limit the development of new methods to address existing evaluation goals, and inappropriate application of commonly-used methods may limit the type of evaluation questions posed, which in turn may slow the progress of our understanding of visualization and its supported processes.

\subsubsection{Many-to-many mapping between scenarios and methods}

Our scenarios do not map directly to a single evaluation method. In fact, many methods are listed under different scenarios. This is due to two reasons. First, we hope by providing a more diverse set of methods in each scenario, we can to encourage evaluators to explore creative ways to employ these methods. One example is the use of the controlled experiment method in Evaluating Visual Data Analysis and Reasoning (VDAR). Given the complexity and open-endedness of VDAR, it may be surprising that a relatively precise and unrealistic method [53] can be used. In our examples, this method was used in the Scented Widget study where the experimenters isolated the factor under investigation [92], and also as part of the evaluation methods in an insight-based study [69].

The second reason for not providing a one-to-one mapping is because we believe that no single evaluation scenario can be exhaustively inspected by one single method in a single evaluation. For example, in Evaluating Collaborative Data Analysis, researchers have used many methods including heuristic evaluations, log analysis, and observations. The variety of evaluation methods found in our scenarios reflects the richness of evaluation opportunities in these scenarios.

\subsubsection{Descriptive, not Prescriptive}

The last point we wish to reflect upon is our decision to take a descriptive rather than prescriptive approach in our project. In other words, we do not assign a certain evaluation method to a goal or question. Rather, we report on studies with similar goals and questions. This is due to our belief that selecting an appropriate evaluation method requires deep understanding of the evaluation goals and constraints. This project is at too high a level to delineate enough details to be prescriptive. Instead, we provide illustrative examples as starting points for evaluators in designing their own studies. 


\begin{tabular}{|c|c|c|c|c|c|c|}
\hline Paper Tags & EuroVis & InfoVis & IVS & VAST & Total & Scenario \\
\hline \multicolumn{7}{|c|}{ Process } \\
\hline 1. People's workflow, work practices & 3 & 1 & 3 & 0 & 7 & UWP \\
\hline 2. Data analysis & 0 & 5 & 3 & 5 & 13 & VDAR \\
\hline 3. Decision making & 0 & 2 & 1 & 4 & 7 & VDAR \\
\hline 4. Knowledge management & 0 & 1 & 0 & 2 & 3 & VDAR \\
\hline 5. Knowledge discovery & 1 & 1 & 0 & 1 & 3 & VDAR \\
\hline 6. Communication, learning, teaching, publishing & 0 & 0 & 4 & 1 & 5 & CTV \\
\hline 7. Casual information acquisition & 0 & 4 & 0 & 0 & 4 & CTV \\
\hline 8. Collaboration & 0 & 3 & 2 & 4 & 9 & CDA \\
\hline \multicolumn{7}{|c|}{ Visualization } \\
\hline 9. Visualization-analytical operation & 0 & 12 & 1 & 0 & 13 & UP \\
\hline 10. Perception and cognition & 17 & 24 & 15 & 3 & 62 & UP \\
\hline 11. Usability/effectiveness & 25 & 84 & 31 & 18 & 158 & UP\&UE \\
\hline 12. Potential usage & 7 & 1 & 5 & 9 & 22 & UE \\
\hline 13. Adoption & 0 & 1 & 3 & 1 & 5 & UE \\
\hline 14. Algorithm performance & 17 & 37 & 15 & 0 & 69 & VA \\
\hline 15. Algorithm quality & 1 & 10 & 12 & 5 & 28 & VA \\
\hline \multicolumn{7}{|c|}{ Not included in scenarios } \\
\hline 16. Proposed evaluation methodologies & 0 & 3 & 0 & 2 & 5 & - \\
\hline 17. Evaluation metric development & 2 & 6 & 1 & 1 & 10 & - \\
\hline
\end{tabular}

TABLE 3

Original coding tags, the number of papers classified, and the final scenario to which they were assigned.

\section{Conclusion}

Our seven evaluation scenarios encapsulate the current state of evaluation practices in our surveyed papers. From the 850 papers we surveyed in the EuroVis, InfoVis, and VAST conferences as well as the IVS journal, we found 361 papers that included evaluations. We coded these evaluations according to seventeen tags (Table 3), condensed these tags into seven scenarios, and classified them into process and visualization.

Scenarios based on process:

- UWP Understanding environments and work practices: to derive design advice through developing a better understanding of the work, analysis, or information processing practices by a given group of people with or without software use.

- VDAR Evaluating visual data analysis and reasoning: to assess how an information visualization tool supports analysis and reasoning about data and helps to derive relevant knowledge in a given domain.

- CTV Evaluating communication through visualization: to assess the communicative value of a visualization or visual representation in regards to goals such as teaching/learning, idea presentation, or casual use.

- CDA Evaluating collaborative data analysis: to understand to what extent an information visualization tool supports collaborative data analysis by groups of people.

Scenarios based on visualization:

- UP Evaluating user performance: to objectively mea- sure how specific features affect the performance of people with a system.

- UE Evaluating user experience: to elicit subjective feedback and opinions on a visualization tool.

- VA Evaluating Visualization Algorithms: to capture and measure characteristics of a visualization algorithm.

These scenarios can be used as a practical context-based approach to exploring evaluation options. To briefly reiterate we provide information on:

1) Choosing a focus: choosing an evaluation focus involves choosing among process or visualization evaluation scenarios and a consideration of the possibly analysis phases.

2) Picking suitable scenarios: scenario in Section 6 give information on evaluation questions and foci.

3) Considering applicable approaches: the scenario descriptions list possible methods and reference examples of where they have been applied.

4) Creating evaluation design and planned analyses: methods and examples provide background literature which can inspire designing evaluation methods for one's own study. However, since evaluation is still in flux, it is important to keep abreast of new evaluation methods in your considerations.

Our scenario approach can, thus, be used as a starting point for expanding the range of evaluation studies and open new perspectives and insights on information visualization evaluation. In contrast to other overview articles 
on evaluation, a major contribution of our work is that we based our evaluation categorization on evaluation questions and goals instead of on existing methods. Our intention is to encourage the information visualization community to reflect on evaluation goals and questions before choosing methods. By providing a diverse set of examples for each scenario, we hope that evaluation in our field will employ a more diverse set of evaluation methods.

For this article, we have coded four main visualization venues and arrived at the codes we used through discussions and several coding passes. We encourage others to extend our coding or to re-code our results at a later point in time to see how the community has evolved in terms of what kind of evaluation papers are published. The full list of papers we coded, together with their respective codes can be found at: http://bit.ly/7-vis-scenarios.

Since our coding is based on the published literature it is entirely possible that further coding can reveal new scenarios and questions which we may not have considered here. We encourage others to publish these findings and help to expand our evolving understanding of evaluation in information visualization, thus developing a repository of examples and scenarios as references for evaluators.

\section{ACKNOWLEDGMENTS}

We would like to thank Adam Perer and Amy Voida for early discussions on structuring evaluation methodologies. We would also like to thank the participants of BELIV 2008 who have contributed material to our early data collection on evaluation methodologies in information visualization.

\section{REFERENCES}

[1] D. L. Alonso, A. Rose, C. Plaisant, and K. L. Norman. Viewing personal history records: a comparison of tabular format and graphical presentation using lifelines. Behaviour \& Information Technology, 17(5):249-262, 1998.

[2] K. Andrews. Evaluation comes in many guises. In CHI workshop on BEyond time and errors: novel evaLuation methods for Information Visualization (BELIV), pages 7-8, 2008.

[3] A. O. Artero, M. C. F. de Oliveira, and H. Levkowitz. Uncovering clusters in crowded parallel coordinates visualizations. In Proceedings of the Symposium on Information Visualization (InfoVis), pages 81-88, Los Alamitos, USA, 2004. IEEE.

[4] K. Baker, S. Greenberg, and C. Gutwin. Heuristic evaluation of groupware based on the mechanicsof collaboration. In Proceedings of Engineering for Human-Computer Interaction, volume 2254 of LNCS, pages 123-139, Berlin, Germany, 2001. Springer Verlag.

[5] L. Barkhuus and J. A. Rode. From mice to men: 24 years of evaluation in chi. In alt.chi: Extended Abstracts of the Conference on Human Factors in Computing Systems (CHI), New York, USA, 2007. ACM.

[6] L. Bartram and C. Ware. Filtering and brushing with motion. Information Visualization, 1(1):66-79, 2002.

[7] B. B. Bederson, B. Shneiderman, and M. Wattenberg. Ordered and quantum treemaps: Making effective use of $2 \mathrm{~d}$ space to display hierarchies. ACM Transactions on Graphics, 21(4):833-854, 2002.

[8] E. Bertini, A. Perer, C. Plaisant, and G. Santucci. Beyond time and errors: Novel evaluation methods for information visualization (beliv). In Extended Abstracts of the Conference on Human Factors in Computing Systems (CHI), pages 3913-3916, New York, USA, 2008. ACM.

[9] I. Brewer, A. M. MacEachren, H. Abdo, J. Gundrum, and G. Otto. Collaborative geographic visualization: Enabling shared understanding of environmental processes. In Proceedings of the Symposium on Information Vizualization (InfoVis), pages 137-141, Los Alamitos, USA, 2000. IEEE.
[10] S. Carpendale. Evaluating information visualizations. In A. Kerren, J. T. Stasko, J.-D. Fekete, and C. North, editors, Information Visualization: Human-Centered Issues and Perspectives, pages 19-45. Springer LNCS, Berlin/Heidelberg, 2007.

[11] C. Chen and Y. Yu. Empirical studies of information visualization: A meta-analysis. International Journal of Human-Computer Studies, 53(5):851-866, 2000.

[12] J. Chen, A. M. MacEachren, and D. J. Peuquet. Constructing overview + detail dendrogram-matrix views. IEEE Transactions on Visualization and Computer Graphics, 15:889-896, 2009.

[13] Y. Chen, L. Wang, M. Dong, and J. Hua. Exemplar-based visualization of large document corpus. IEEE Transactions on Visualization and Computer Graphics, 15:1161-1168, 2009.

[14] R. K. Christopher, C. G. Healey, V. Interrante, D. H. Laidlaw, and C. Ware. Thoughts on user studies: Why, how, and when. IEEE Computer Graphics and Applications, 23:2003, 2003.

[15] J. Corbin and A. Strauss. Basics of Qualitative Research: Techniques and Procedures for Developing Grounded Theory. Sage Publications, Thousand Oaks, CA, USA, 2008.

[16] L. Costello, G. Grinstein, C. Plaisant, and J. Scholtz. Advancing user-centered evaluation of visual analytic environments through contests. Information Visualization, 8:230-238, 2009.

[17] J. W. Creswell. Research Design. Qualitative, Quantitative, and Mixed Methods Approaches. Sage Publications, Inc., Thousand Oaks, CA, USA, $2^{\text {nd }}$ edition, 2002.

[18] M. P. Czerwinski, M. V. Dantzich, G. Robertson, and H. Hoffman The contribution of thumbnail image, mouse-over text and spatial location memory to web page retrieval in $3 \mathrm{~d}$. In Proceedings of INTERACT, pages 163-170. IOS Press, 1999.

[19] J. Drury and M. G. Williams. A framework for role-based specification and evaluation of awareness support in synchronous collaborative applications. In Proceedings of the Workshops on Enabling Technologies: Infrastructure for Collaborative Enterprises (WETICE), pages 12-17, Los Alamitos, USA, 2002. IEEE.

[20] T. Dwyer and D. R. Gallagher. Visualising changes in fund manager holdings in two and a half-dimensions. Information Visualization, 3 (4):227-244, 2004.

[21] R. Eccles, T. Kapler, R. Harper, and W. Wright. Stories in geotime. Information Visualization, 7(1):3-17, 2008.

[22] G. Ellis and A. Dix. An exploratory analysis of user evaluation studies in information visualization. In Proceedings of the AVI Workshop on BEyond time and errors: novel evaLuation methods for Information Visualization (BELIV), 2006.

[23] L. Grammel, M. Tory, and M.-A. Storey. How information visualization novices construct visualizations. IEEE Transactions on Visualization and Computer Graphics, 16:943-952, 2010.

[24] S. Greenberg and B. Buxton. Usability evaluation considered harmful (some of the time). In Proceedings of the Conference on Human Factors in Computing Systems (CHI), pages 217-224, New York, USA, 2008. ACM.

[25] S. Greenberg, G. Fitzpatrick, C. Gutwin, and S. Kaplan. Adapting the locales framework for heuristic evaluation of groupware. Australian Journal of Information Systems (AJIS), 7(2):102-108, 2000.

[26] S. Greenberg. Observing collaboration: Group-centered design. In T. Erickson and D. W. McDonald, editors, HCI Remixed: Reflections on Works That Have Influenced the HCI Community, chapter 18, pages 111-118. MIT Press, Cambridge, USA, 2008.

[27] J. Grudin. Why cscw applications fail: Problems in the design andevaluation of organizational interfaces. In Proceedings of the Conference on Computer-Supported Cooperative Work (CSCW), pages 85-93, New York, USA, 1988. ACM.

[28] C. Gutwin and S. Greenberg. The mechanics of collaboration: Developing low cost usability evaluation methods for shared workspaces. In Proceedings of the Workshops on Enabling Technologies: Infrastructure for Collaborative Enterprises (WETICE), pages 98-103. IEEE Computer Society, 2000.

[29] M. Hao, D. A. Keim, U. Dayal, D. Oelke, and C. Tremblay. Density displays for data stream monitoring. Computer Graphics Forum, 27 (3):895-902, 2008.

[30] J. Heer and M. Bostock. Crowdsourcing graphical perception: Using mechanical turk to assess visualization design. In Proceedings of the Conference on Human Factors in Computing Systems (CHI), pages 203-212, New York, USA, 2010. ACM.

[31] J. Heer and danah boyd. Vizster: Visualizing online social networks. In Proceedings of the Symposium on Information Visualization (InfoVis), pages 33-40, New York, USA, 2005. ACM.

[32] J. Heer and G. Robertson. Animated transitions in statistical 
data graphics. IEEE Transactions on Visualization and Computer Graphics, 13(6):1240-1247, 2007.

[33] J. Heer, F. B. Viégas, and M. Wattenberg. Voyagers and voyeurs: Supporting asynchronous collaborative information visualization. In Proceedings of the Conference on Human Factors in Computing Systems (CHI), pages 1029-1038, New York, USA, 2007. ACM.

[34] D. M. Hilbert and D. F. Redmiles. Extracting usability information from user interface events. ACM Computing Survey, 32(4):384-421, 2000

[35] U. Hinrichs, H. Schmidt, and S. Carpendale. EMDialog: Bringing information visualization into the museum. IEEE Transactions on Visualization and Computer Graphics, 14(6):1181-1188, 2008.

[36] K. Holtzblatt and S. Jones. Contextual Inquiry: A Participatory Technique for Systems Design. Lawrence Earlbaum, Hillsdale, NJ, USA, 1993

[37] K. Hornbæk and E. Frokjær. Reading of electronic documents: The usability of linear, fisheye and overview+detail interfaces. In Proceedings of the Conference on Human Factors in Computing Systems (CHI), pages 293-300, New York, USA, 2001. ACM.

[38] P. Isenberg, A. Bezerianos, N. Henry, S. Carpendale, and J.-D. Fekete. CoCoNutTrix: Collaborative retrofitting for information visualization. Computer Graphics and Applications: Special Issue on Collaborative Visualization, 29(5):44-57, 2009

[39] P. Isenberg, D. Fisher, M. Ringel Morris, K. Inkpen, and M. Czerwinski. An exploratory study of co-located collaborative visual analytics around a tabletop display. In Proceedings of the Conference on Visual Analytics Science and Technology (VAST), pages 179-186, Los Alamitos, USA, 2010. IEEE.

[40] P. Isenberg, A. Tang, and S. Carpendale. An exploratory study of visual information analysis. In Proceeding of the Conference on Human Factors in Computing Systems (CHI), pages 1217-1226, New York, USA, 2008. ACM.

[41] P. Isenberg, T. Zuk, C. Collins, and S. Carpendale. Grounded evaluation of information visualizations. In Proceedings of the $\mathrm{CHI}$ Workshop on BEyond time and errors: novel evaLuation methods for Information Visualization (BELIV), pages 56-63, New York, USA, 2008. ACM.

[42] M. Y. Ivory and M. A. Hearst. The state of the art in automating usability evaluation of user interfaces. ACM Computing Surveys, 33 (4):470-516, 2001.

[43] F. Janoos, S. Singh, O. Irfanoglu, R. Machiraju, and R. Parent. Activity analysis using spatio-temporal trajectory volumes in surveillance applications. In Proceedings of the Symposium on Visual Analytics Science and Technology (VAST), pages 3-10, Los Alamitos, USA, 2007. IEEE.

[44] Y.-a. Kang, C. Görg, and J. Stasko. Evaluating visual analytics systems for investigative analysis: Deriving design principles from a case study. In Proceedings of the Symposium on Visual Analytics Science and Technology (VAST), pages 139-146, Los Alamitos, USA, 2009. IEEE

[45] A. Kittur, E. H. Chi, and B. Suh. Crowdsourcing user studies with mechanical turk. In Proceeding of the Conference on Human Factors in Computing Systems (CHI), pages 453-456, New York, USA, 2008. ACM.

[46] O. Kulyk, R. Kosara, J. Urquiza, and I. Wassin. Human-computered aspects. In G. Goos, J. Hartmanis, and J. van Leeuwen, editors, Lecture Notes in Computer Science, pages 13-76. Springer, 2007.

[47] H. Lam and T. Munzner. Increasing the utility of quantitative empirical studies for meta-analysis. In Proceedings of the $\mathrm{CHI}$ Workshop on BEyond time and errors: novel evaLuation methods for Information Visualization (BELIV), pages 21-27, New York, USA, 2008. ACM.

[48] H. Lam, R. A. Rensink, and T. Munzner. Effects of 2d geometric transformations on visual memory. In Proceedings of the Symposium on Applied Perception in Graphics and Visualization (APGV), pages 119-126, New York, USA, 2006. ACM.

[49] J. D. Mackinlay, P. Hanrahan, and C. Stolte. Show me: Automatic presentation for visual analysis. IEEE Transactions on Visualization and Computer Graphics, 13(6):1137-1144, 2007.

[50] N. Mahyar, A. Sarvghad, and M. Tory. A closer look at note taking in the co-located collaborative visual analytics process. In Proceedings of the Conference on Visual Analytics Science and Technology (VAST), pages 171-178, Los Alamitos, USA, 2010. IEEE.

[51] R. Mandryk. Modeling User Emotion in Interactive Play Environments: A Fuzzy Physiological Approach. PhD thesis, Simon Fraser University, 2005.

[52] G. Mark and A. Kobsa. The effects of collaboration and system transparency on CIVE usage: An empirical study and model. Presence, 14(1):60-80, 2005

[53] J. McGrath. Methodology matters: Doing research in the behaviora and social sciences. In Readings in Human-Computer Interaction: Toward the Year 2000. Morgan Kaufmann, 1994.

[54] T. Munzner. A nested process model for visualization design and validation. IEEE Transactions on Visualization and Computer Graphics, 15(6):921-928, 2009.

[55] D. C. Neale, J. M. Carroll, and M. B. Rosson. Evaluating computersupported cooperative work: Models and frameworks. In Proceedings of the Conference on Computer-Supported Cooperative Work (CSCW), pages 112-121, New York, USA, 2004. ACM.

[56] W. Peng, M. O. Ward, and E. A. Rundensteiner. Clutter reduction in multi-dimensional data visualization using dimension reordering. In Proceedings of the Symposium on Information Visualization (InfoVis), pages 89-96, Los Alamitos, USA, 2004. IEEE.

[57] A. Perer and B. Shneiderman. Integrating statistics and visualization for exploratory power: From long-term case studies to design guidelines. IEEE Computer Graphics and Applications, 29(3):3951, 2009.

[58] D. Pinelle and C. Gutwin. A review of groupware evaluations. In Proceedings of the Workshops on Enabling Technologies: Infrastructure for Collaborative Enterprises (WETICE), pages 86-91, Los Alamitos, USA, 2000. IEEE.

[59] D. Pinelle and C. Gutwin. Groupware walkthrough: Adding context to groupware usability evaluation. In Proceedings of the Conference on Human Factors in Computing Systems (CHI), pages 455-462, New York, USA, 2002. ACM.

[60] D. Pinelle, C. Gutwin, and S. Greenberg. Task analysis for groupware usability evaluation: Modeling shared-workspace tasks with the mechanics of collaboration. ACM Transactions on Human Computer Interaction, 10(4):281-311, 2003.

[61] P. Pirolli and S. Card. The sensemaking process and leverage points for analyst technology as identified through cognitive task analysis. In Proceedings of International Conference on Intelligence Analysis, 2005.

[62] C. Plaisant, J. Grosjean, and B. Bederson. Spacetree: Supporting exploration in large node link tree, design evolution and empirical evaluation. In Proceedings of the Symposium on Information Visualization (InfoVis), pages 57-64, Los Alamitos, USA, 2002. IEEE.

[63] C. Plaisant. The challenge of information visualization evaluation. In Proceedings of the Working Conference on Advanced Visual Interfaces (AVI), pages 109-116, New York, USA, 2004. ACM.

[64] Z. Pousman, J. Stasko, and M. Mateas. Casual information visualization: Depictions of data in everyday life. IEEE Transactions on Visualization and Computer Graphics, 13(6):1145-1152, 2007.

[65] A. J. Pretorius and J. J. van Wijk. Visual inspection of multivariate graphs. Computer Graphics Forum, 27(3):967-974, 2008.

[66] G. Robertson, M. Czerwinski, K. Larson, D. C. Robbins, D. Thiel, and M. van Dantzich. Data mountain: Using spatial memory for document management. In Proceedings of the Symposium on User Interface Software and Technology, pages 153-162, New York, USA, 1998. ACM

[67] A. Robinson. Collaborative synthesis of visual analytic results. In Proceedings of the Symposium on Visual Analytics Science and Technology (VAST), pages 67-74, Los Alamitos, USA, 2008. IEEE.

[68] J. Sajaniemi and M. Kuittinen. Visualizing roles of variables in program animation. Information Visualization, 3(3):137-153, 2004.

[69] P. Saraiya, C. North, and K. Duca. An evaluation of microarray visualization tools for biological insight. In Proceedings of the Symposium on Information Visualization (InfoVis), pages 1-8, Los Alamitos, USA, 2004. IEEE.

[70] P. Saraiya, C. North, V. Lam, and K. A. Duca. An insight-based longitudinal study of visual analytics. Transactions on Visualization and Computer Graphics, 12(6):1511-1522, 2006.

[71] K. Sedig, S. Rowhani, J. Morey, and H.-N. Liang. Application of information visualization techniques to the design of a mathematical mindtool: A usability study. Information Visualization, 2(3):142159,2003

[72] M. Sedlmair, D. Baur, S. Boring, P. Isenberg, M. Jurmu, and A. Butz. Requirements for a mde system to support collaborative in-car communication diagnostics. In CSCW Workshop on Beyond the Laboratory: Supporting Authentic Collaboration with Multiple Displays, 2008.

[73] M. Sedlmair, P. Isenberg, D. Baur, and A. Butz. Information visualization evaluation in large companies: Challenges, experiences and recommendations. Information Visualization Journal, 10(3), 
2011

[74] J. Seo and B. Shneiderman. Knowledge discovery in highdimensional data: Case studies and a user survey for the rank-byfeature framework. Transactions on Visualization and Computer Graphics, 12(3):311-322, 2006.

[75] W. Shadish, T. Cook, and D. Campbell. Experimental and QuasiExperimental Designs. Houghton Mifflin Company, 2002.

[76] B. Shneiderman and C. Plaisant. Strategies for evaluating information visualization tools: Multi-dimensional in-depth long-term case studies. In Proceedings of the AVI workshop on BEyond time and errors: novel evaluation methods for information visualization (BELIV), New York, USA, 2006. ACM.

[77] B. Shneiderman and C. Plaisant. Designing the User Interface: Strategies for Effective Human-Computer Interaction. AddisonWesley, $5^{\text {th }}$ edition, 2009.

[78] T. Skog, S. Ljungblad, and L. E. Holmquist. Between aesthetics and utility: Designing ambient information visualizations. In Proceedings of the Symposium on Information Visualization (InfoVis), pages 233240, Los Alamitos, USA, 2003. IEEE Computer Society.

[79] H. Song, E. Curran, and R. Sterritt. Multiple foci visualisation of large hierarchies with flextree. Information Visualization, 3(1):1935, 2004.

[80] G. Stahl. Group Cognition. MIT Press, 2006.

[81] G. Symon. Qualitative Research Diaries, pages 94-117. Sage Publications, 1998.

[82] J. Thomas and K. A. Cook, editors. Illuminating the Path: The Research and Development Agenda for Visual Analytics. IEEE Computer Society Press, 2005.

[83] M. Tory and T. Möller. Evaluating visualizations: Do expert reviews work? Computer Graphics and Applications, 25(5):8-11, 2005

[84] M. Tory, D. W. Sprague, F. Wu, W. Y. So, and T. Munzner. Spatialization design: Comparing points and landscapes. IEEE Transactions on Visualization and Computer Graphics, 13(6):12621285,2007

[85] M. Tory and S. Staub-French. Qualitative analysis of visualization: A building design field study. In Proceedings of the CHI Workshop on BEyond time and errors: novel evaLuation methods for Information Visualization (BELIV), New York, USA, 2008. ACM.

[86] J. G. Trafton, S. S. Kirschenbaum, T. L. Tsui, R. T. Miyamoto, J. A. Ballas, and P. D. Raymond. Turning pictures into numbers: Extracting and generating information from complex visualizations. International Journal of Human-Computer Studies, 53(5):827-850, 2000

[87] M. Twidale, D. Randall, and R. Bentley. Situated evaluation for cooperative systems. In Proceedings of the Conference on ComputerSupported Cooperative Work (CSCW), pages 441-452, New York, USA, 1994. ACM.

[88] Usability.net. Usability.net methods. Website, 2009. http://www. usabilitynet.org/tools/methods.htm.

[89] F. Viégas, E. Perry, E. Howe, and J. Donath. Artifacts of the presence era: Using information visualization to create an evocative souvenir. In Proceedings of the Symposium on Information Visualization (InfoVis), pages 105-111, Los Alamitos, USA, 2004. IEEE.

[90] F. Viégas, M. Wattenberg, F. van Ham, J. Kriss, and M. McKeon. Many Eyes: A site for visualization at internet scale. IEEE Transactions on Visualization and Computer Graphics, 12(5):1121-1128, 2007.

[91] C. Weaver, D. Fyfe, A. Robinson, D. Holdsworth, D. Peuquet, and A. M. MacEachren. Visual exploration and analysis of historic hotel visits. Information Visualization, 6(1):89-103, 2007.

[92] W. Willett, J. Heer, and M. Agrawala. Scented widgets: Improving navigation cues with embedded visualizations. IEEE Transactions on Visualization and Computer Graphics, 12(5):1129-1136, 2007.

[93] K. Wongsuphasawat and B. Shneiderman. Finding comparable temporal categorical records: A similarity measure with an interactive visualization. In Proceedings of the Symposium on Visual Analytics Science and Technology (VAST), pages 27-34, Los Alamitos, USA, 2009. IEEE.

[94] B. Yost and C. North. The perceptual scalability of visualization. IEEE Transactions on Visualization and Computer Graphics, 13(6): 837-844, 2007.

[95] T. Zuk, L. Schlesier, P. Neumann, M. S. Hancock, and S. Carpendale. Heuristics for information visualization evaluation. In Proceedings of the AVI Workshop on BEyond time and errors: novel evaLuation methods for Information Visualization (BELIV), pages 55-60, New York, USA, 2006. ACM.

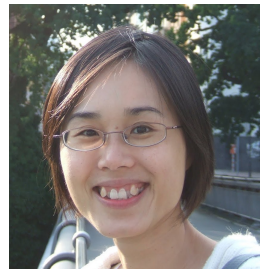

Heidi Lam Heidi Lam is a Software Engineer at Google Inc. Heidi received her Ph.D. from the University of British Columbia in 2008. Her main research interests include information visualization with the focus of exploratory data analysis, and evaluations in information visualization.

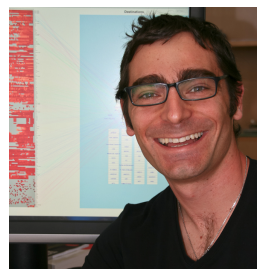

Enrico Bertini Dr. Enrico Bertini is a research associate at the University of Konstanz, Germany. He obtained his PhD from Sapienza University of Rome, Italy, in 2006 with a thesis on clutter reduction in information visualization. His main research interest is visual analytics, with a specific focus on large high-dimensional data, integration of automatic and interactive data analysis, and evaluation.

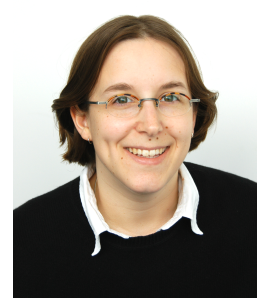

Petra Isenberg Petra Isenberg is a research scientist at INRIA, Saclay, France in the Aviz research group. Prior to joining INRIA, Petra received her $\mathrm{PhD}$ from the University of Calgary in 2009 and her Diplom-degree in Computational Visualistics from the University of Magdeburg in 2004. Her main research area is information visualization and visual analytics with a focus on collaborative work scenarios. She is interested in exploring how people can most effectively work together when analyzing large and complex data sets on novel display technology such as touch-screens or tabletops.

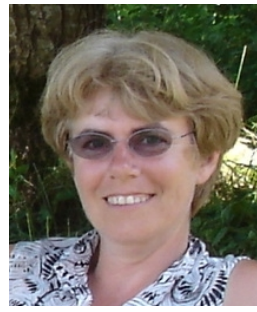

Catherine Plaisant Dr. Catherine Plaisant is Senior Research Scientist at the HumanComputer Interaction Laboratory of the University of Maryland Institute for Advanced Computer Studies. She earned a Doctorat d'Ingnieur degree in France in 1982 and joined HCIL in 1987. She enjoys most working with multidisciplinary teams on designing and evaluating new interface technologies that are useable and useful. She has written over 100 refereed technical publications on the subjects of information visualization, evaluation methods, electronic health record interfaces, digital libraries, online help, etc. She co-authored with Ben Shneiderman the 5th Edition of Designing the User Interface. 


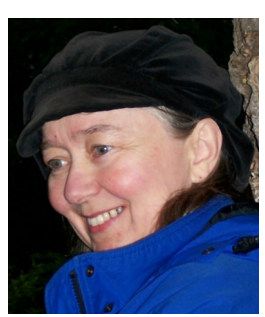

Sheelagh Carpendale Sheelagh Carpendale is a Professor at the University of Calgary where she holds a Canada Research Chair in Information Visualization and an NSERC/iCORE/SMART Industrial Research Chair in Interactive Technologies. She directs the Innovations in Visualization (InnoVis) research group and Computational Media Design, an interdisciplinary graduate degree specialization. Her research on information visualization, large interactive displays, and new media art draws on her dual background in Computer Science (BSc., Ph.D. Simon Fraser University) and Visual Arts (Sheridan College, School of Design and Emily Carr, College of Art). 


\section{APPENDIX A}

\section{TAGS USED IN OPEN CODING}

We developed our seven scenarios based on the following 17 tags. These tags were used to open code publications from four venues. The distribution of publication by tags and venue is listed in Table 3.

1) Data analysis: Evaluate how visualization is used in exploratory data analysis to generate hypotheses.

2) Decision making: Evaluate how visualization is used to confirm or refute solutions to problems or hypotheses.

3) Collaboration: Evaluate how visualization supports collaboration activities.

4) Adoption: Observe how a visualization is adopted after deployment.

5) Communication, learning, teaching, publishing: Study how visualization is used in multiple forms of communication activities.

6) Usability/Effectiveness: Solicit usability feedback or determine effectiveness of visualization based on user performance.

7) People's workflow, work practices: Understand potential users' work environment and practices.

8) Perception and cognition: Study low-level human perception and cognition to evaluate or explore the visualization design space.

9) Algorithm performance: Study efficiency and performance of the algorithm that renders the visualization.

10) Knowledge discovery: Study how the visualization supports knowledge discovery.

11) Potential usage: Solicit users' opinions on how the visualization may be useful.

12) Proposed Evaluation Methodologies: Propose new methodologies on evaluation.

13) Visualization-analytical operation: Study how visualization affects users' performance of simple visual tasks.

14) Casual information acquisition: Study how users casually acquire information, especially in ambient displays.

15) Evaluation metrics development: Propose new evaluation metrics.

16) Algorithm quality: Evaluate algorithms when compared to accepted gold standards such as human judgments.

17) Knowledge management: Evaluate how effectively does the system support management of knowledge generated in the sense-making loop. 


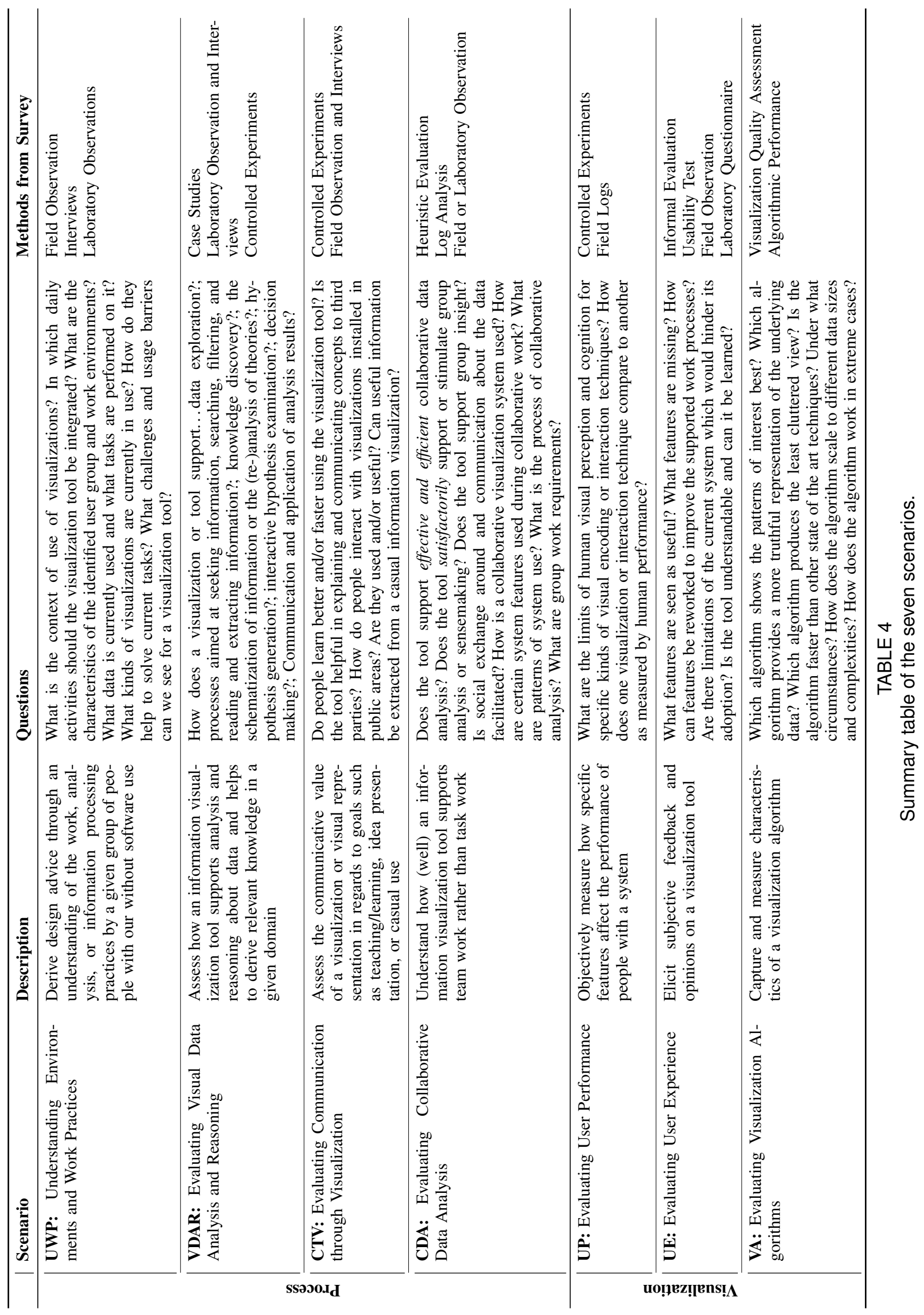

